\title{
Presencia de los agradecimientos en los artículos científicos en revistas médicas nacionales y extranjeras
}

\author{
ESTHER RAMÍREZ GODOY \\ DirecciónG eneraldeBiblio tecas \\ de la UNAM, 04510, México D.F., \\ E-mail:eramirez@ selene.cichcu.unam.mx \\ JANE M. RuSSELL \\ CentroUniversitariodeInvestigacionesBibliotecológicas \\ delaUNAM, 04510, México D.F., Tel: 56- 23- 03- 29. \\ E-mail:jrussell@ servidor.unam.mx
}

\begin{abstract}
RESUMEN
Enel presenteestudio seanalizaron lascatego ríaspro puestasporCro nin en 1991: Redibuaón; Apoyo Moral; Auxiliares Ténicos, Impulsor Pinipal; AsesarA cedtadb, paraanalizarlosagradecimien tospresentesen lasrevistasmédicasnacio nalesyextranjeras.Losresultadosindicaronunamayor presen ciadelasec ción deagradecimientosen revistasex tranjerasqueen las nacionales y patrones distintos con respecto a las categorías de Cronin. La categorías de Auxiliaresy de A poyoMoral fueron las más fre cuentes en revistas nacionales y con respecto a las revistas extranjeras éstas fueron las de Asesor Aceeditadby de Impulsar Primipal. Consideramos que los resultados encontrados podrían explicarse, por lo menos en parte, por diferencias en los contextos científicos.

Pa labras clave: Agra de cimientos, Revis tas Médicas, México, Adeu do In telectual
\end{abstract}

\section{THE ROLE OF ACKNOWLEDGEMENTS IN RESEARCH ARTI CLES PUBLISHED IN NATIONAL AND FOREIGN MEDICAL JOURNALS E STHER RAMÍREZ-GODOY JANE M. RUSSELL}

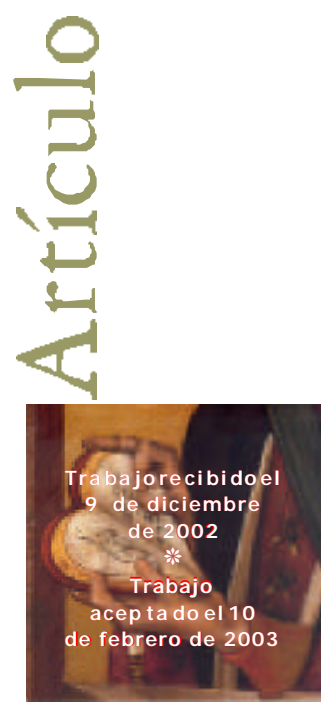

\begin{abstract}
In the present study we used the categories proposed by Cronin in 1991 (Paymaster,MoralSupport;Dagsbody; Tednical;PrimeMove; TrustedAssessa) to analyzetheacknowledgementsfound innatio nal and fo reignmedicaljour nals. Resultsshowed agreaterpresen ce of acknowledge mentsin thein ter national rather than the national publications and distinct patterns with respect to the different categories. Dossoody and Maral Suppot were the most common categories in the national journals and Trusted Assessorand PrimeMovewith res pect to the fo reign jour nals. We suggest that the diffe rences in scientific contexts could go a long way to explaining results. Key Words: Acknowledge ments, Me di cal Jour nals, Me xi co, In te llec tual Debt
\end{abstract}




\section{INTRODUCCIÓN}

Tos agradecimientos en los trabajos científicos comparten ciertas características

$ـ$ funcionales y textuales con las citas, las cuales pueden ser visualizadas desde perspectivas múltiples y analizadas desde diversos enfoques (Mackintosh, 1972; Cro nin, 1991).Losagradecimientospueden operarcomo medicio nesdeunaverda dera asesoría (Chubin, 1975), ocultarin fluen cia(Cro nin 1992) o manifestarcrédito o recompensa (Mackintosh, 1972). También pueden verse como intercambio de cumplidos (McCain, 1991), como artificios de ordenamiento o "arreglo", como sinopsis, pies de nota o referen cias(BenAri, 1987), como apén dicesritualísticos(Cronin,1991), o como expresio nesdeso lidaridad característicosdeloscam posacadémi cos denominados por escuelas o sistemas de enseñanza bien organizados (Ben Ari, 1987). Por su parte Katz (1985) men cio naqueel agradecimien to sirvecomo fun ción científica, ya que los temas sobre ciencia toman significado de su contexto histórico tan to como desusméritosin trínsecos;elagradecimien to co lo caal trabajo decadacual directamente en el contexto de su comunidad científica inmediata, y la sección de agradecimientos es una manifestación histórica de los esfuerzos realizados por la ciencia.

En cam posquetienen un alto grado defac tualismo in telectual o ideo ló gico (por ejemplo enfilo so fíapolítica, antropolo gíacultural,lingüística, críticaliteraria, etcéte ra), losagradecimien tospueden usarseparaen viarmeta- men sajestalescomo: "pertenezco a este gru po" o "sosten go estafe". D eunaformamás ge neral, losagradeci mientos pueden emplearse junto con la bibliografía maestra o lista de referencias vin culadasaunartículo publicado, amodo delo calizaral au torenunambien teparticularcognoscitivo yso cial, en estecaso elmeta- men sajees: "éstaeslacom pañíadela quemerodeo".

En1991Cro nin inició unaseriedeestu diosparaex plo rarlafun ción so cialyel signi ficado cognoscitivo delosagradecimien tosylosagrupó enseiscatego rías-Reribuioón; A poyoMaral; Auxiliares, Ténicos, Impulsor Prinipal; Asesar Aceeitadb Propuso además que los agradecimientos y las citas se utilizaran en formacon jun taparaevaluarel de sempeño del científico pero ad virtió quesibien lascitasylosagradecimien tostienen ciertascaracterísticasen co mún, no hayqueprecisaro forzarlo análo go:eltípico trabajo académico conlleva, pormo tivosdeargu men to un pro medio de 20 citas, pero sólo uno decadadostrabajosdecorteacadémico llevaunagradecimien to.Lasdiferen cias de población son en ton cesasun tosqueradican en órdenesdenivelesdemagnitud,lo cualno significaquelosagradecimientosno seansignosdeatencióncrítica.

Elanálisisdecitastienequeverconlosdatosquedescriben mo dosformalesdecomunicaciónacadémica:laspublicacionessereferen cian,lafrecuenciadecitasseregistraderiván do sedeunavariedad demedicio nesdel im pacto,lacuentadecitasleotorga alanalistaunmedio convenien te- sibienim perfecto - decalibrarlainfluen ciayla eminen cia.Lacitaesnatural menteun pro ceso so cial (Cro nin, 1991):unmecanismo institucionalizado para otorgarles crédito y reconocimiento a los pares. Se supone que la 
cita de un autor por parte de otro es usualmente significativa, porque tiene relación con un resul tado im portan te;ymien trasmáscitasregistradas existan en favordeun autor, mayoressuin fluen ciayel prestigio queselepresume, pero decual quierforma elnúmero decitas esun regis tro in com pleto delain fluen ciaso cial, un pun to queno hasido deltodo igno rado; "lacien ciano hadesarro llado mecanismospararecono cer la influencia en los trabajos, excepto para el ocasional 'comunicación personal'” y "agradecimientos" que habitualmen teno están in cluidasen losdatosqueto man en cuentalosanalistasdecitas." [MacRo bertsyMacRo berts1989]

La comparaciónagradecimiento/ citaes impulsada por un conjunto pequeño de individuos altamente receptores de agradecimientos y pudiera ser que estos individuosmuyagradecidossean abundantementecitadoscuan do elconjun to queotorga elagradecimien to estáacon sideración, pero tam bién pueden existirau to resmuyci tadosqueno reciban agradeci mien tos. D ecual quierformano haymodo prác tico de compro barestahipó tesisen ausen ciadeuníndicedeagradecimientoscom parableal delascitas.

Sinembargo haysuficientesargumentosparaadmitirquelosagradecimientosson mo dosdeestablecerran gosco rrelativosdeestatusdecitas(porejem plo,juiciospor pares)quepueden usarseen ejerciciosdeevaluación institu cio nal, o en lospro cesos relacio nadosconlo gros/ pro mo ción como unin dicadoradicio naldeim pacto, méri to o influen ciapercibida. Pero hastaqueseclarifiquenlasnormasdelagradecimiento yseanimealosauto resanormalizarsu com portamien to al respecto, el pleno po tencial de estos artefactos textuales largamente olvidados no alcanzará plenamente su valor.Peseaqueel hecho deagradeceres, enlarealidad, un pro ceso altamen tepersonal espoco pro bablequesu cum baapresio nes deestetipo.Partedel valorybon dad delagradecimien to radicaensunaturaleza, amenudo muypersonaleidiosincrática, y espro bablequeopongaresisten ciatanto alanormalizacióncomoalamecanización.

Losagradecimientosgeneralmenteno seincluyen enlosdatosconloscualeslaboraelanalistadecitas(MacRo bertsandMacRo berts, 1989;Mullins, 1973), po dríaser revelador conocer la medidaen quela ex clu sión deagradecimien tos del pro ceso de revisiónacadémicahacedistin ción en treo con traaquelloscuyainfluen ciaseregistra o reflejademaneras distintaso complemen tariasalapublicación,ycitacionessubsiguientes. El asunto po dríavolverseverdaderamentemásinteresan tesilospro pó si tos discutidos por los Institutos Nacionales de Salud en los EUA se adoptaran ampliamente. De acuerdo con Tiller (1990) los institutos nacionales de salud, en un esfuerzo porcontenerlaprácticacrecientedeenlistaravecesdo cenasdeauto res, ha sugerido un "sistema graduado" que acreditaría a los investigadores principales como au to res, con otrosalos cualesselesbrin daríaagradecimien to como con tribur yen tes; sital pro pó sito seadop tael significado so cialdelagradecimien to po dríamodificarsedrásticamente.

Lacon tribución deunasesoracreditado, unárbitro anó nimo o un consejero doctoral no siempre se reflejará vía una cita, pero sería valioso considerar que: "cada científico pro ductivo tieneporlo generalalgunosaseso resacreditadosqueno están 
en su departamen to o labo rato rio yquecon frecuen ciano tienen lamismaespecialidad ni a veces la misma disciplina, yloscualesfun gen como críticosamigables dela obra del autor 0 , más precisamente, de su labor". Estos científicos de asesores no compartennecesariamentealosmismosasesoresacreditadosyamenudonisiquiera tienen uno de ellos en común (Evered and Harnett, 1989). En algunos aspectos el agradecimiento que acom pañaal escrito final es el equivalen tealacita (en términos reales), pero no tieneelpesoyelvalordeestaúl tima.

D adalaim portan ciavin culadaalapublicaciónycuen tadecitacionesenlaevalua ción del desempeño de investigación a nivel individual, institucional o nacional (Edge,1979), esdealgunaformaanó malo queelagradecimien to seexcluyadelacontabilidadacadémica. Ser men cio nado enlasec ción deagradecimien tos esdealguna maneracon denarseal lim bo, puesel estatuspreciso deun agradecimien to aún no es claro del todo. En el sistemaderecom pen sasdelacien cia, el solo agradecimien to indicainfluen ciadelacon tribuciónintelectualrecono cida, pero no harecibido unt rato equivalentealacita;indepen dientementedecuán trivial,negativao su perficialseala cita, se presume que es más significativa(en términos de deuda intelectualmedible) que él más elogioso agradecimiento, suposición que es lógicamente inconsistente y empíricamenteatacable.

"Un salón delafama" cons trui do sólo con citas esun edificio cuestio nable, lainfluen ciaacadémicaesalgo queseexhibeyejerceunagran variedad demo delos, al gur nosmenossutilesyevidentesquelacita:actividad educativa, mentoría, tuto ría, y ase so ría, en treotros. Ko chen (1987) plan teaunain terro gan te ¿quétan gran dedebeser la deuda para implicar una cita? Pero también cabe cuestionarse por el lado de los agradecimien tos, pueshastalafechaelartedeagradecerpermanecemuyinex plo ra do en el pro ceso deco municación primaria: ¿quétan persuasivaesestaprác tica? ¿si gueelfraseo delagradecimien to un estilo particular? ¿cuáleselsignificado so cial del agradecimiento? ¿̇ego biernaelagradecimiento porunconjuntotácito denormas?

Mu cho delo quesehadichoyanalizado respecto al com portamien to so brelacita ción po dríaigual men teaplicarseenlo to can tealosagradecimien tos.D eacuerdo con Mackintosh (1991) la carencia de interés en los agradecimientos, no indica que sea precisamenteescasasurelevan ciaen losreco no cimien tosdelacien cia, 0 , siesasí, enton ces, lascitas queuno recibedesu trabajo porpartedeotro caen den tro del mismo estilo. Las tesis doctorales, prólogos de monografías y codas de artículos de revista con frecuen ciallevan agradeci mien tos deuno u otro tipo cuidado samen teparafra seadoso redactados pero se han hecho po cosin ten tos poranalizarel significado socialdeestepro ceso ritualizado yubicarlo en laam pliaestructuradel sistemadereconocimientoscientíficos.

Sielagradecimien to vaatratarsecomo unin dicadordelflujo in telectual, o como co ordenadaquemapealosvín culosdeco municacióninformalden tro o en trelasdis ciplinas, enton cesseríaim portantedistinguirclaramentedesdeel prin cipio entrelas motivacionesquesehallandetrásdelosdiferentestiposdeagradecimientos. 
Se reconoce el papel fundamental que juega la publicación científica como instrumentoválido paraevaluarlainvestigacióncientíficanacionalein ternacio nal:vehículo detransmisión delco no cimien to; recurso esen cialparalaen señanza; medio deco mu nicación so brelosnuevoshallaz gos; mecanismo dedivul gación delascien cias, su his to ria, susideasysusavan ces; medio dedefinición ydifu sión delvo cabulario cien túfico $\mathrm{y}$, finalmente,como pro mo tordeldesarro llo científico ein dicadordelacienciaque se produce.Sabido es tam bién quelasec ción deagradecimien tosseen cuen traen trelos requerimientos uniformes de normalización para publicar escritos científicos. Pero hastaelmo mento no existenin gúnreporteanivelnacionalacercadeestasección.

D etodo lo an teriorsurgió elin terés porilus trarcon datosel rolquedesem peñala sección deagradecimientoscomo apo yo enlainvestigación mexicana. Unavezplanteadalaim portan ciadeestu diarel agradecimien to en el con tex to nacio nal, sepro cedió en el presen teestu dio aselec cio narelárea. Porunlado México esun paísfértilen historia expresada a través detex tos, yun com plemen to in dispen sabledetodo esto es la investigación médica nacional. Ambas partes (historia e investigación médica) consolidan un gran bagaje cultural que enriquece enormemente la investigación cien tíficaquesedesarro llahoyen día. Porelotrolarazón paraelegireláreamédicaes quedeto daslasáreasdelacien ciaylatecno lo gíacul tivadasenAméricaLatina, lainvestigaciónmédicaeslaquetienemayortradiciónylamásproductiva.Pruebadeello esqueamediados del siglo pasado estaáreadabacuen tadecasilami tad delas publicacionescientíficasgeneradaspormexicanosenrevistasextranjeras.D isciplinas como labio química, lafisio lo gía, labio lo gíacelu larylasneuro cien cias, alcanzaron los más altos índices de citas que la comunidad científica mundial le dedicó a trabajos realizados en el país (Martínez Palomo 1994). En 1994 el área más desarrollada, la quepro dujo elmayornúmero detrabajosen revistasespecializadasylaquegeneró el mayornúmero decitas, siguiósien dolainvestigaciónmédica.

Portan to el objetivo del presenteestudio esanalizarycaracterizarelpapelquedesempeñan los agradecimientos en las revistasmédicasmexicanas y com pararlocon suscontrapartesextranjeras.

\section{Procedimiento}

Para realizar este estudio se analizaron los trabajos publicados en siete revistas médicasnacionales( Cuadro1)yen16revistasmédicasextranjeras(Cuadr2).

Losdatosdelasrevistasestudiadasfueronobtenidosenlasdirecciones:

* http:/ / gateway1.ovid.com:80/ ovidweb.cgi

http:/ / nlmpubs.nlm.nih.gov/ online/ journals/ ljiweb.pdf

Elperio do aevaluarfueel trans currido deenero de1986adiciem brede1996. El grupo deestasrevistasfueseleccionado porcontarconlassiguientessemejanzas:

a) Pertenecer al área médica

b) Tener una periodicidad regular 
c) Contar con su disponibilidad física o en línea en las bibliotecas de la Ciudad de México,

d) Estar basado en los resultados obtenidos por la tesis de licenciatura de Angélica Ro sasyMaríaInésEscalan te(1995), quienesiden tificaron aéstas como lasrevis tasquemásartículospublicaron deau to resmexicanosenelperio do 1981-1985.

El caadro3in dicaelto tal deartículosanalizadosporaño tanto en lasrevistasnacionalescomo en las ex tranjeras. Enal gunasrevistasno fuepo siblerealizarelanálisis de todos los fascículos correspondientes al periodo de estudio. Sin embargo en todos loscasoselanálisiscubrió el 80\% delosnúmerosaparecidosde1986a1996. Seregis trólapresen ciao ausen ciadeagradecimientosenlosartículosestudiados.Paraanalizarlosartículoscontenidosen las revistasan tesmen cio nadas, seaplicaron loscriteriosdelesquemaoriginaldeCronin(1991)( Cuadro4).

Laevaluación delasrevistastan to nacio nalescomo extranjerasselimitóalosartículos originales, yseex cluyeron del estudio o trasseccio nescomo edito riales, cró nicas, cartas al edi tor, etcétera, asícomo otros tiposdeartículos:relatosdecasosclínicos, notas o comentarios clínicos, ensayos, reuniones bibliográficas, descripciones de instrumentos nuevos, descripciones completas de una enfermedad y monografías, entreotrasmás.

\section{RESULTADOS}

\section{Presencia de los agradecimientos}

El aadro5desglo saporañolosporcentajesobtenidosenlas7revistasmédicasna cionalesencuantoalapresenciadelaseccióndeagradecimientos.

D elto taldeartículoscontenidosenlassieterevistasmédicasnacionalesdurante el perío do quevade1986a1996, so lamen teel $24 \%$ in cluíalasec ción deagradecimientos. La presencia de esta sección en el perio do 1986-1996esno tablementevariable. Sin embargo se puede observar que el año de 1988 alcanzó el porcentaje más bajo (13\%) derepresen tación deestasec ción. En cam bio elmayorporcen tajedepresenciadeestasec ción lo ob tu vieron los años 1992y 1996; un 29\%.D e1990 en adelan te selo calizanlosmayo resporcentajesdepresenciadelaseccióndeagradecimientos, lo cual podría significar, por un lado, un incremento en los apoyos prestados y, por el otro,laexisten ciademayorconcienciadeparticipacióndelosinvestigado resso bre la obligación dereco no cerelapoyo prestado.Lasrevistasquerepresentan elmayoryel menorporcen tajedelapresen ciadeesta sección son:ArdhivosdalnstitutodbCardiolo ǵa, con el 42\% yArchivosdelnvestigacónMédica, con el 12\%. Larevis taArdhivosdelnves tigacónMédicaapareció in dexadaen el ScienceCitationIndex has ta el año de 1992, despuésdejó defigurarporunlapso deseisañosyreapareció en el1998.

D el to taldelosartículoscontenidosenlas16revistasmédicasextranjerasdurante el perio do de1986a1996, el 47\% presen taron lasec ción deagradecimien tos. Elcuar dro6 desglosa los porcentajes obtenidos por año. El año de 1986 dio el porcentaje 
másbajo detodo el perio do analizado, represen tado porel 32\%. Porsu parteel año de mayor porcentaje en relación con la presencia de esta sección fue 1992, con el $55 \%$.Lasrevistasqueal can zaron el másalto yel más bajo porcen tajedepresen ciade lasección deagradecimien tosfueron ClinicalReserch, con el 57\%, yAmericanjarnalof Mediane, con un 32\%.

Loscuadros $5 y 6$ muestran al gu nos añoscon un porcen tajede " 0 ", lo quesedebe a que la revistadedicó su publicacióna:homenajesainvestigadores,instituciones 0 fundaciones(importantesparaesarevista)memorias, conferenciasyaniversarios.

\section{D istribución de las seis categorías de agradecimientos}

En el aadro7semuestranlosresultadosdelas7revistasmédicasnacio nalesylas6 catego ríasdeagradecimien to queCro nin 1991 in cluyeen su esquemac1 $=$ Retribur ción, $\mathrm{c} 2=$ Apo yo mo ral, $\mathrm{c} 3=$ A uxiliar, $\mathrm{c} 4=$ Técnico, $\mathrm{c} 5=$ = Im pulsorprin cipalyc6 = Asesoracreditado.

Enorden descen dentelosresul tadosfueron lossiguien tes:27\% paralacatego ría de "Auxiliar"; $25 \%$ para "A po yo Mo ral"; $21 \%$ para "Retribu ción"; $14 \%$ para "Ase sorAcreditado";12\% para"Técnico",yfinalmente2\% para "ImpulsorPrincipal”.

Elcuadro 8muestraqueel 31\% sein clinahacialacatego ríade "AsesorAcredita do", mien trasque "Im pulsorPrin cipal" estárepresentado con un $25 \%$; con un $12 \%$ la de "Auxiliar"; $11 \%$ lascatego ríasde "Técnico yA poyo Mo ral" y, final men telade másbajoporcentajefue "Retribución",conun $10 \%$.

Lafigura1 in cluyelasdiferen ciasen trelosám bitosdepublicacióntan to nacio nal como extranjera con respecto a las categorías de agradecimiento más reconocidas porlosauto resdeacuerdo conlaayu daprestadaporin dividuos, organizacio nesy/ o fundaciones.

\section{DisCUSIÓN}

A pesardelaexisten ciadeunalargatradición cien tíficamexicanaen eláreamédi ca,losresultadosobtenidosen elpresenteestudio in dican quelaco munidadnacional agradece poco la ayu daprestadatraslacul minación deun trabajo.Pruebadeello es quedelosartículosanalizadosenlassieterevistasmédicasnacio nalesseleccio nadas únicamenteel $24 \%$ in cluyelasec ción deagradecimientos. Enelcaso delas 16 revistas médicasextranjeras, elporcentajefueeldoble.

Cabemen cio narquedelasseis catego rías en lasquefueron evaluadoslosagrade cimientosemitidosporlosauto resdelasrevistasnacio nales, losdostipos másreferidos fueron los relacionados con el apoyo Auxiliar (c3=27\%) y el Apoyo Moral (c2=25\%). El apoyo Auxiliar se refiere al tipo de apoyo secretarial, los consejos en cuan to alabor edito rial ylaayu daen lacap tu radedatos, yel A po yo Mo ralsevincula con el apoyo institucional, el acceso a instalaciones, el uso de equipo, y el apoyo en tiempo. D e acuerdo con los resultados obtenidos el tipo de apoyo que agradecenlos 
autores que publican en lassieterevistasnacio nales, serefiereaun apo yo tan gible 0 lo gístico.Enesteaspecto Cro nin(1991) catalo gaalas catego ríasc2yc3,como aque llasqueestánrelacionadasconlosrecursosyprocedimientos, respectivamente.

Porotro lado laevaluación delasseiscatego rías deagradecimien tosenlosartícur lospublicadosen revistas extranjeras, mostró quelosdostiposdeagradecimiento de mayorporcentajefueron, porunaparte, lacatego ríadeA sesorAcreditado (c6=31\%) relacionado con la retroalimentación, el análisisylos co mentarioscríticosdepares, co labo rado resyco legasqueactúan como portavo cesau to rizadosyfuen tesdenue vas opinio neso ideas; yporlaotraladeIm pul sorPrin cipal(c5=25\%), quein vo lu cra la inspiración o un estímulo otorgado por el investigador principal, el director del pro yec to o elasesordetesis. Porotraparte, en losresultados ob tenidosenlaevaluación de las revistas extranjeras se percibelapresen ciadelas catego ríasc5yc6 en las queel tipo deapo yo esmás deorden delo in telec tual.Cro nin (1991) catalo gaaestas catego ríascomoapoyosdetipo conceptual.

Existenvariosfacto resquepudieronhaberintervenido enlosresultadosobtenidos en el presente estudio. Hablar acerca de artículos publicados en revistas nacionales y ex tranjerasim plicaporsupuesto men cio narquesetratadedoscon tex tosto talmente diferentesenloscualeslosinvestigado ressedesenvuelvenydesarrollansusestudios.

Por una parteexisten al gu nossu puestosacercadelainexisten ciadelasec ción de agradecimien tos en losartículosmédicos, el primero deloscualestocaeltemadela éticaylamo ral, temasquepueden haberrepercutido en losresultadosobtenidosen este estudio. Haciendo un análisis retrospectivo relacionado con el tema de la "ética”, se identificó que en los años 70 Rodríguez Sala de G ómezgil (1977) realizó un trabajo en el quemuestralascaracterísticasbásicasquetodo buen cien tífico debereunir,entrelascualessecuen tan buenamemo ria, inven tivaycapacidad deanálisis; sin em bargo no hacereferen ciaalaim portan ciadetenerunalto sen tido deéticaymo ral queesesen cial en el cien tífi co. D eigual formaen 1991 Fortesy Lom nitz en su libro so brelaformacióndelcientífico en México describenlascaracterísticasdelcientífico ideal, pero sorpresivamente la ética no formaba parte de los requisitos básicos que todo cien tífico ideal debepo seer. 0 tro pun to es el relacio nado con lasnormaspara los autores que pretenden publicaren revistasmédicas, lascualessuelen lo calizarse en lacon traportadadecadanúmero delarevistaycuyo contenido seapegaalasN or mas de Vancouver (In ternatio nalCommittee of Medical JournalE ditors2001). Se gúnlosresultados ob tenidosen unestudio previo (Ramírez G o doy, 1994),lasrevis tas médicas nacionales no presentan una descripción detallada de cada una de las seccionesqueconformanlasnormasparalosauto res. Encambiolainformaciónque sedaen las revistasmédicas ex tranjeras en relación con estemismo pun to essu ma menteam plia, puesdefinecadaunadelasseccio nesypresentalascaracterísticaspropias decadasec ción, asícomo laim portan ciaquetienecadaunadeellas, in cluyen do loconcernientealaseccióndeagradecimientos.

Unaterceraconsideración paraqueun artículo no in cluyalasección deagradeci mientos simplemente pueda atribuirse a que el autor trabajó solo y con recursos 
eco nó micosymaterialespro piosdesuinstitu ción, yqueporlo tan to no tienelanecesidad deutilizarestasección. Sin em bargo cadadíasonmenoslos trabajosdeinvesti gación queno requieren dein sumosex ternosalosrecursospro piosdelosinvestiga dores del proyecto, y la retroalimentaciónde los colegas, el préstamo de equipo o el donativo dereactivosentreotros(Silva,1990), pueden serayudasesenciales.

Los estudios previos indican marcadas diferencias entre los campos por lo que toca al peso y las característicasdelosagradecimientosenlasrevistasprincipales, lo cual sepo dríaex ten deranivel deespecialidadesenáreascomo lamedicina. McCain (1991) basó suanálisisen unarevistaex tran jeradeláreadelagenéticayen con tró que el $59 \%$ deartículosin cluyen agradecimien tospormaterialbio ló gico facilitado,y que un porcentaje similar lo hace por "consejos y discusiones realizados con colegas 0 porcomentariosconrespecto almanuscrito". Enpsico lo gía, so cio lo gía, histo riayfilo so fíaCro ninysusco labo rado res 1993 en con traron queun $90.8 \%, 88.0 \%, 65.1 \%$ y $37.5 \%$, respectivamente, deartículoscontabanconporlomenosunagradecimiento. Elestudio deCro nin aplicó unatipo logíamo dificadadelplanteamiento originalpara que la categoría más representadaen todos estos cuatro campos (rango de $78.1 \%$ a 95.5\% delto taldelosagradecimien tos)fuerade "co municacióninteractivaentrepa res" ydeestemodo serelacionaraconlascategoríasc5yc6desuesquemaoriginal.

Lapocapresen ciadeagradecimien tos detipo "asesoracreditado o im pulsorprincipal" enlasrevistasmédicasnacio nales, hacepoco práctico con tem plareldesarrollo deindicado resdeadeudo in telectual paracom plementarelreco no cimien to através deunanálisisdecitasen lospro cesosnacio nalesdeevaluación del desem peño científico. Sin contar con información fidedigna que explique el comportamiento de los investigadores en los diferentes cam posalahoradereco no ceren formaex plícitala ayu dadetipo con cep tual prestadaporco legas, cual quieranálisismétrico en estesentido seríatan solo unaapro ximaciónalaverdad. Sin embargo, como reco no cen otros autores, elestudio cuan titativoycualitativo delosagradecimien tosenrevistas es pe cializadasen distintoscam posesunaformaderevelaradeudosintelectualesentreespecialistas, quedeotro modo pueden quedarocul tos den tro delasredesdeco muni caciónconocidascomoloscolegiosinvisibles.

\section{BiBLIOG RAFÍA.}

Ben Ari, E. "On acknowledgements in ethnographies". Jaumal of Anthropdogical Reserrch (1987), 43: 63-84.

Chu bin, D .E. "Trusted assessorship in scien ce: arelation in need of data" . Social Studies of Saiene (1975), 5: 362-368.

Cro nin, B. "TheCitationProcess TheRdeandSignificancefCitationinSäntific Commmication". London: Taylor G raham, (1984). 
Cro nin, B. "Letthecreditsroll: apreliminaryexamination of therolepla yed bymen torsand trustedassessorsin disciplinaryformation". Jaumal ofDcoumentation (1991), 47: 227-239.

Cro nin, B. "Thehidden in fluen cers: an examination of theroleplayed by mentorsand trusted assessorsin theevo lution of informationscience". In: Vakkari, P. and Cronin, B. eds. Conceptions of LibraryandInfomation Saience Histarical, Empirical and Theretical Pespeetives London: Taylor Graham, (1992), 126-134.

Cronin,B., McKenzie, G. and Rubio, L. "The norms of acknowledgements in four humanities and social sciences disciplines". ". Joumal of Doumetation, (1993). 49: 29-43.

Edge, D. "Q uantitative measures of communication in scien ce:acritical review". Histary of Saiene (1979), 17: 102-134.

Evered, D . and Hamett, S., eds. "TheEvaluation of SäentificReserden". Chichester: Wiley, (1989).

Fortes, J. y Lomnitz, L. "La formacón da dentíficoen Méxicoadquiriendbuna nueva idantidad”. México: Siglo Veintiuno Editores, (1991), p.113-120.

Internatio nal Committee of MedicalJournalE ditors. "Uniformequirements for manuscipts submitted to biomedical joumals". (2001) [citado, 4 de julio 2002]. Disponible en

http:/ / www.acponline.org/ journals/ resource/ unifreqr.htm

Katz, M.J. "Elements of a Säentific Paper". New Haven: Y ale University Press, (1985), p. 80.

Kochen, M. "How well do we acknowledge intellectual debts?" Jamal of Doumentation (1987), 43: 54-64.

Mackintosh, K.H. "Acknowledgements patterns in sociology". Unpublished PhD dissertation. University of O regon, 1972. Citado en:Cronin, B. "Let thecreditsroll:apreliminary examination of theroleplayer by mentors and trusted assessors in disciplinary formation". Jaumal of Doumetation (1991), 47: 227-239.

MacRo berts, M.H.and MacRo berts, B.R. "Pro blems of citation analysisa critical review". Jamal of the American Socidy for Infomation Saience, (1989), 40: 342-349.

McCain, K.W. "Communication, competition and secrecy: the production and dissemination of research related information in genetics". Säene, TedndogyandHumanValues (1991), 16: 491-516. 
Martínez Palomo, A. "El desarrollocontemparánœedelas ȧenias dela saluden Méxiø”. En: Méxi co:Cien ciayTec no lo gíaen el Um bral del Si glo XX I. México: CONACYT. 1994. p. 171-214.

Mullins, N.C. "Theories and Theary Groups in Contemporary Ameican Sociology'. London: Harper and Row, (1973), p.18.

Ramírez Godoy, M.E. "El artículo científico en las ciencias biomédicas editado por la UNAM: su normalización estructural". 1994. Tesis (bibliotecología). México: Universidad Nacional Autónoma deMéxico, FaaultaddeFilosofíayLetras CdeejodeBiblidtedogóa p. 68-79.

Rodríguez Sala de Gómezgil, M.L. "El CientíficoenMéxica SuImagenetre los Estudiantes deEnseñanza Media”. México:Universidad Nacio nalA ut tónoma de México, (1977), p. 176.

RosasGutiérrez,A.M.yEscalan teVargas, M.I. "Lapro ducción científica mexicanaen revistascientíficasnacio naleseinternacionalesatravésde las bases de datos PERIÓ DICA y BLAT (1981-1985)". 1995. Tesis (Bibliotecología). México: Univesidad Naaional Autónma deMéxic, FaaltaddeFilosofía y Letras CdegjodeBildidtedogóap. 138-142.

Silva, G .A. "La autoría múltiple y la autoría injustificada en los artículos científicos" BdeíndeSanidad- Panamá, (1990), 108: 136.

Tiller, B.C. "Medical databases and health information systems" In: Williams, M.E., ed. Annual Reviewof Infomation Saiereand Teehndogy, 25. Washington: Elsevier/ ASIS, 1990, p. 313-382. 
Presencia de los agradecimientos en los artículos científicos en revistas... 171

Cuadro 1

Característicasde las Revistas Nacionales Seleccionadas para el Presente Estudio

\begin{tabular}{|c|c|c|c|c|c|c|}
\hline Título & $\begin{array}{c}\text { Disciplina } \\
\text { Medline }\end{array}$ & Tipo de & Frecuencia & Tiraje & Idioma & $\begin{array}{l}\text { 1er. Año de } \\
\text { publicación }\end{array}$ \\
\hline $\begin{array}{l}\text { Archivos Insitito } \\
\text { deCardidoǵa }\end{array}$ & $\begin{array}{c}\text { Cardiología } \\
*\end{array}$ & $\begin{array}{c}\text { Instituto de in- } \\
\text { vestigación }\end{array}$ & Bimestral & 2,500 & $\begin{array}{c}\text { Texto Inglés } \\
* * * \text { T. C. español }\end{array}$ & 1930 \\
\hline $\begin{array}{c}\text { Ardivosdelnves } \\
\text { tigaáón Mética }\end{array}$ & $\begin{array}{c}\text { Cien cias Mé } \\
\text { dicas } \\
* *\end{array}$ & \begin{tabular}{|c|} 
Instituto \\
Mexicanodel \\
Seguro Social \\
\end{tabular} & Trimestral & 1,900 & Texto inglés & 1971 \\
\hline $\begin{array}{l}\text { Bdé́n Médico } \\
\text { Hospitallnfantil } \\
\text { deMéxico }\end{array}$ & $\underset{* *}{\text { Pediatría }}$ & $\begin{array}{l}\text { Hospital de } \\
\text { gobierno }\end{array}$ & Mensual & 3,500 & $\begin{array}{l}\text { Texto y***T.C. } \\
\text { inglésespañol }\end{array}$ & 1944 \\
\hline $\begin{array}{l}\text { Gacta Méica } \\
\text { México }\end{array}$ & $\begin{array}{l}\text { Medicina } \\
\quad * \\
\end{array}$ & $\begin{array}{l}\text { Asociación } \\
\text { profesional }\end{array}$ & Bimestral & 20,000 & $\begin{array}{l}\text { Textoespañol } \\
\text { ***T.C. inglés } \\
\end{array}$ & 1864 \\
\hline $\begin{array}{c}\text { Reista deInvesti- } \\
\text { gadónClínica }\end{array}$ & $\begin{array}{c}\text { Medicina } \\
*\end{array}$ & \begin{tabular}{|c|}
$\begin{array}{c}\text { Hospital de go- } \\
\text { bierno }\end{array}$ \\
\end{tabular} & Bimestral & 1,800 & $\begin{array}{l}\text { Texto y ***T.C. } \\
\text { inglés/ español }\end{array}$ & 1948 \\
\hline $\begin{array}{l}\text { Reista Médica dd } \\
\text { IMSS (Institito } \\
\text { Mexicamodd } \\
\text { Seguro Social) } \\
\end{array}$ & $\begin{array}{l}\text { Ciencias } \\
\text { médicas } \\
* *\end{array}$ & $\begin{array}{c}\text { Instituto Mexi } \\
\text { cano del Segut } \\
\text { ro Social }\end{array}$ & Bimestral & 2,800 & $\begin{array}{l}\text { Tex to in glés/ } \\
\text { español }\end{array}$ & 1971 \\
\hline $\begin{array}{l}\text { SaludPública } \\
\text { México }\end{array}$ & $\begin{array}{c}\text { Salud pública } \\
*\end{array}$ & $\begin{array}{c}\text { Instituto de in- } \\
\text { vestigación }\end{array}$ & Bimestral & 4,000 & $\begin{array}{l}\text { Textoespañol } \\
\text { ***T.C. inglés }\end{array}$ & 1959 \\
\hline
\end{tabular}

* $\quad$ List of Journals Indexed in Index Medicus/ MED LINE

** Ulrich's

*** Tabla de Contenido

**** Science Citation Index

***** Social Sciences Citation Index 
172 InvestigacónBiblicteedógica v. 16 N o. 33 julio/ diciembre de 2002

Cuadro 2

Características de las Revistas ExtranjerasSeleccionadas para el PresenteEstudio

\begin{tabular}{|c|c|c|c|c|c|c|c|}
\hline Título & $\begin{array}{l}\text { Disciplina } \\
\text { MEDLINE }\end{array}$ & $\begin{array}{l}\text { Tipo de } \\
\text { Institución }\end{array}$ & Frecuencia & Tiraje & País & Idioma & $\begin{array}{l}\text { 1er. Año de } \\
\text { Publicación }\end{array}$ \\
\hline $\begin{array}{l}\text { Ameican } \\
\text { *J. of Mediane }\end{array}$ & Medicina & Comercial & **18 veces & 53,751 & USA & Inglés & 1946 \\
\hline $\begin{array}{l}\text { Ardivesof } \\
\text { Andrdogy }\end{array}$ & $\begin{array}{l}\text { Endocrinología } \\
\text { Reproducción }\end{array}$ & Comercial & **3veces & 50,800 & $\begin{array}{l}\text { Reino } \\
\text { Unido }\end{array}$ & Inglés & 1978 \\
\hline $\begin{array}{l}\text { Ardivesof } \\
\text { IntemalMediane }\end{array}$ & Medicina interna & $\begin{array}{l}\text { Asociación } \\
\text { profesional }\end{array}$ & $* * 22$ veces & 999,000 & USA & Inglés & 1908 \\
\hline $\begin{array}{l}\text { Brain } \\
\text { Research }\end{array}$ & $\begin{array}{l}\text { Psiquiatría } \\
\text { Neurología }\end{array}$ & Comercial & Mensual & 66,780 & Holanda & Inglés & 1966 \\
\hline $\begin{array}{l}\text { Clinical } \\
\text { Research }\end{array}$ & $\begin{array}{l}\text { Farmacia } \\
\text { Farmacología }\end{array}$ & $\begin{array}{l}\text { Asociación } \\
\text { profesional }\end{array}$ & Mensual & 56,900 & $\begin{array}{l}\text { Reino } \\
\text { Unido }\end{array}$ & Inglés & 1977 \\
\hline Contraception & Reproducción & Comercial & Mensual & 48,750 & USA & Inglés & 1970 \\
\hline Gastroentedogy & G astroenterología & $\begin{array}{l}\text { Asociación } \\
\text { profesional }\end{array}$ & Mensual & 45,860 & USA & Inglés & 1978 \\
\hline Hepatdoy & G astroenterología & $\begin{array}{l}\text { Asociación } \\
\text { profesional }\end{array}$ & Mensual & 5,718 & USA & Inglés & 1981 \\
\hline *J. of Bacteidogy & Bacteriología & $\begin{array}{l}\text { Asociación } \\
\text { profesional }\end{array}$ & Quincenal & 6,970 & USA & Inglés & 1916 \\
\hline *J. of Detistry & O dontología & Comercial & $* * 8$ veces & 8,567 & $\begin{array}{l}\text { Reino } \\
\text { Unido }\end{array}$ & Inglés & 1972 \\
\hline $\begin{array}{l}\text { *J. of Natural } \\
\text { Produts Lloyda }\end{array}$ & $\begin{array}{c}\text { Farmacia } \\
\text { Biología } \\
\text { Química }\end{array}$ & $\begin{array}{l}\text { Asociación } \\
\text { profesional }\end{array}$ & Mensual & 2,300 & USA & Inglés & 1938 \\
\hline $\begin{array}{l}\text { *J. of } \\
\text { Rhemmatdogy }\end{array}$ & Reumatología & $\begin{array}{l}\text { Asociación } \\
\text { profesional }\end{array}$ & Mensual & 3,500 & USA & Inglés & 1974 \\
\hline $\begin{array}{l}\text { Merical } \\
\text { Hypotheses }\end{array}$ & Medicina & $\begin{array}{l}\text { Asociación } \\
\text { profesional }\end{array}$ & Mensual & 300 & $\begin{array}{l}\text { Reino } \\
\text { Unido }\end{array}$ & Inglés & 1975 \\
\hline $\begin{array}{l}\text { NewEngand } \\
\text { *J. of Meeliaine }\end{array}$ & Medicina & $\begin{array}{l}\text { Asociación } \\
\text { profesional }\end{array}$ & Semanal & 55,588 & USA & Inglés & 1978 \\
\hline $\begin{array}{l}\text { Phamacdogy, Bio } \\
\text { dhemistryand } \\
\text { Bdhavior }\end{array}$ & Farmacología & Comercial & Mensual & 8,960 & USA & Inglés & 1973 \\
\hline $\begin{array}{l}\text { Physiologyand } \\
\text { Bdhavior }\end{array}$ & Psicología & Comercial & **15 veces & 1,800 & USA & Inglés & 1966 \\
\hline
\end{tabular}

$\begin{array}{ll}* & \text { Journal } \\ \text { ** ( ) veces al año }\end{array}$ 
Presencia de los agradecimientos en los artículos científicos en revistas... 173

Cuadro 3

Número de artículos en revistas nacionales y extranjeras analizados por año

\begin{tabular}{|c|c|c|c|c|c|c|c|c|c|c|c|c|}
\hline \multicolumn{12}{|c|}{ Periodo1986-1996 } & \multirow[t]{2}{*}{ Total } \\
\hline Año & 1986 & 1987 & 1988 & 1989 & 1990 & 1991 & 1992 & 1993 & 1994 & 1995 & 1996 & \\
\hline Art. Nac. & 240 & 291 & 307 & 288 & 289 & 302 & 392 & 266 & 299 & 281 & 212 & 3,167 \\
\hline Art.Inter. & 86 & 91 & 85 & 88 & 88 & 104 & 99 & 107 & 105 & 109 & 104 & 1,066 \\
\hline Total & 326 & 382 & 392 & 376 & 377 & 406 & 491 & 373 & 404 & 390 & 316 & 4,233 \\
\hline
\end{tabular}

Cuadro 4

Ca te go rías de agra de cimien tos pro pues tos por Cronin 1991

\begin{tabular}{|l|l|}
\hline \multicolumn{1}{|c|}{ Categoría } & \multicolumn{1}{|c|}{ Tipo de Apo yo } \\
\hline Retribución(c1) & Becas, subsidios, apoyo con material. \\
\hline $\begin{array}{l}\text { Apoyo } \\
\text { Mo ral (c2) }\end{array}$ & $\begin{array}{l}\text { Apoyoinstitucional, acceso a instalaciones, } \\
\text { uso de equipo, apo yo con tiempo, acceso a } \\
\text { conjun to dedatos, etc. }\end{array}$ \\
\hline Auxiliar (c3) & $\begin{array}{l}\text { Apoyo secretarial, con sejosen cuan to alala } \\
\text { bor edito rial, ayu daen captura de datos, ac- } \\
\text { cesoyanálisis. }\end{array}$ \\
\hline Técnico (c4) & $\begin{array}{l}\text { Consejos en cuanto a programación, acceso } \\
\text { a conocimientostécnicos, ayu daen pro cedi } \\
\text { mientosestadísticos. }\end{array}$ \\
\hline $\begin{array}{l}\text { Impulsor } \\
\text { Principal (c5) }\end{array}$ & $\begin{array}{l}\text { Inspiración o im pul sos dadosporelinvesti } \\
\text { gadorprincipal, directordel proyecto, asesor } \\
\text { detesis. }\end{array}$ \\
\hline $\begin{array}{l}\text { Asesor } \\
\text { Acreditado (c6) }\end{array}$ & $\begin{array}{l}\text { Retroalimentación, análisis y comentarios } \\
\text { críticos de pares,colaboradores y colegas } \\
\text { queactúan como portavo cesautorizados y } \\
\text { fuentes de nuevasopinio neso ideas. }\end{array}$ \\
\hline
\end{tabular}


Cuadro 5

Presencia de la sección de agradecimientos en los artículos publicados en las revistas médicas nacionales

Periodo 1986-1996

\begin{tabular}{|c|c|c|c|c|c|c|c|c|c|c|c|c|}
\hline $\begin{array}{l}\text { Nom bre de la } \\
\text { Revista }\end{array}$ & 1986 & 1987 & 1988 & 1989 & 1990 & 1991 & 1992 & 1993 & 1994 & 1995 & 1996 & Total \\
\hline $\begin{array}{l}\text { Ardhivos dd Insti- } \\
\text { tutodeCardidoǵa }\end{array}$ & $52 \%$ & $28 \%$ & $26 \%$ & $21 \%$ & $31 \%$ & $62 \%$ & $75 \%$ & $35 \%$ & $35 \%$ & $47 \%$ & $45 \%$ & $42 \%$ \\
\hline $\begin{array}{l}\text { Ardivos deInvesti- } \\
\text { gadónMédica }\end{array}$ & $0 \%$ & $14 \%$ & $12 \%$ & $7 \%$ & $11 \%$ & $10 \%$ & $15 \%$ & $17 \%$ & $24 \%$ & $10 \%$ & $16 \%$ & $12 \%$ \\
\hline $\begin{array}{l}\text { Bdeín Mécico- } \\
\text { Hospital Infantilde } \\
\text { México }\end{array}$ & $30 \%$ & $20 \%$ & $19 \%$ & $0 \%$ & $8 \%$ & $15 \%$ & $10 \%$ & $14 \%$ & $11 \%$ & $17 \%$ & $19 \%$ & $15 \%$ \\
\hline $\begin{array}{l}\text { Gacta Médica } \\
\text { México }\end{array}$ & $0 \%$ & $13 \%$ & $0 \%$ & $40 \%$ & $39 \%$ & $21 \%$ & $27 \%$ & $18 \%$ & $13 \%$ & $26 \%$ & $20 \%$ & $20 \%$ \\
\hline $\begin{array}{l}\text { Revista deInvesti } \\
\text { gacoón Clínica }\end{array}$ & $37 \%$ & $27 \%$ & $21 \%$ & $47 \%$ & $28 \%$ & $29 \%$ & $30 \%$ & $54 \%$ & $35 \%$ & $30 \%$ & $42 \%$ & $35 \%$ \\
\hline $\begin{array}{l}\text { Revista Médica - } \\
\text { IMSS }\end{array}$ & $15 \%$ & $27 \%$ & $11 \%$ & $10 \%$ & $25 \%$ & $16 \%$ & $16 \%$ & $25 \%$ & $5 \%$ & $20 \%$ & $14 \%$ & $17 \%$ \\
\hline $\begin{array}{l}\text { SaludPública } \\
\text { México }\end{array}$ & $0 \%$ & $8 \%$ & $0 \%$ & $14 \%$ & $25 \%$ & $34 \%$ & $30 \%$ & $23 \%$ & $44 \%$ & $36 \%$ & $50 \%$ & $24 \%$ \\
\hline Totales & $19 \%$ & $21 \%$ & $13 \%$ & $20 \%$ & $24 \%$ & $27 \%$ & $29 \%$ & $27 \%$ & $24 \%$ & $27 \%$ & $29 \%$ & $24 \%$ \\
\hline
\end{tabular}


Presencia de los agradecimientos en los artículos científicos en revistas... 175

\section{Cuadro6}

Presencia de la sección de agradecimientos en los artículos publicados en revistas médicas extranjeras

Periodo 1986-1996

\begin{tabular}{|c|c|c|c|c|c|c|c|c|c|c|c|c|}
\hline $\begin{array}{l}\text { Nom bre de la } \\
\text { Revista }\end{array}$ & 1986 & 1987 & 1988 & 1989 & 1990 & 1991 & 1992 & 1993 & 1994 & 1995 & 1996 & Total \\
\hline $\begin{array}{l}\text { Ameican Jaumal } \\
\text { of Mediane }\end{array}$ & $0 \%$ & $43 \%$ & $42 \%$ & $0 \%$ & $0 \%$ & $46 \%$ & $47 \%$ & $47 \%$ & $44 \%$ & $41 \%$ & $38 \%$ & $32 \%$ \\
\hline $\begin{array}{l}\text { Andhivesof } \\
\text { Andrdogy }\end{array}$ & $56 \%$ & $0 \%$ & $73 \%$ & $63 \%$ & $76 \%$ & $43 \%$ & $71 \%$ & $52 \%$ & $66 \%$ & $53 \%$ & $72 \%$ & $57 \%$ \\
\hline $\begin{array}{l}\text { ArdhivesfInternal } \\
\text { Mediäne }\end{array}$ & $30 \%$ & $41 \%$ & $28 \%$ & $32 \%$ & $38 \%$ & $35 \%$ & $30 \%$ & $32 \%$ & $62 \%$ & $62 \%$ & $46 \%$ & $40 \%$ \\
\hline Brain Restarch & $32 \%$ & $48 \%$ & $52 \%$ & $63 \%$ & $59 \%$ & $54 \%$ & $63 \%$ & $67 \%$ & $72 \%$ & $63 \%$ & $36 \%$ & $55 \%$ \\
\hline Clinical Research & $50 \%$ & $38 \%$ & $72 \%$ & $64 \%$ & $56 \%$ & $48 \%$ & $68 \%$ & $58 \%$ & $44 \%$ & $62 \%$ & $72 \%$ & $57 \%$ \\
\hline Contraception & $15 \%$ & $53 \%$ & $48 \%$ & $33 \%$ & $57 \%$ & $62 \%$ & $57 \%$ & $53 \%$ & $73 \%$ & $62 \%$ & $52 \%$ & $51 \%$ \\
\hline Gastroenterdogy & $38 \%$ & $52 \%$ & $68 \%$ & $52 \%$ & $38 \%$ & $55 \%$ & $48 \%$ & $52 \%$ & $55 \%$ & $70 \%$ & $0 \%$ & $48 \%$ \\
\hline Hepatdogy & $52 \%$ & $44 \%$ & $32 \%$ & $48 \%$ & $32 \%$ & $62 \%$ & $56 \%$ & $48 \%$ & $32 \%$ & $62 \%$ & $43 \%$ & $46 \%$ \\
\hline $\begin{array}{l}\text { Jaumal of } \\
\text { Bacteidogy }\end{array}$ & $0 \%$ & $52 \%$ & $67 \%$ & $52 \%$ & $56 \%$ & $48 \%$ & $67 \%$ & $48 \%$ & $32 \%$ & $52 \%$ & $48 \%$ & $47 \%$ \\
\hline Jaumal ofDentistry & $48 \%$ & $71 \%$ & $32 \%$ & $54 \%$ & $48 \%$ & $68 \%$ & $48 \%$ & $56 \%$ & $68 \%$ & $38 \%$ & $68 \%$ & $54 \%$ \\
\hline $\begin{array}{l}\text { Jaumal of Natural } \\
\text { Produds_Lloydia }\end{array}$ & $38 \%$ & $0 \%$ & $42 \%$ & $62 \%$ & $32 \%$ & $62 \%$ & $38 \%$ & $62 \%$ & $42 \%$ & $38 \%$ & $0 \%$ & $38 \%$ \\
\hline $\begin{array}{l}\text { Jaumal of } \\
\text { Rhamatdogy }\end{array}$ & $38 \%$ & $63 \%$ & $57 \%$ & $57 \%$ & $48 \%$ & $62 \%$ & $62 \%$ & $57 \%$ & $41 \%$ & $44 \%$ & $65 \%$ & $54 \%$ \\
\hline MedicalHypotheses & $0 \%$ & $38 \%$ & $63 \%$ & $23 \%$ & $61 \%$ & $33 \%$ & $45 \%$ & $51 \%$ & $38 \%$ & $23 \%$ & $41 \%$ & $38 \%$ \\
\hline $\begin{array}{l}\text { NewEngandJaur- } \\
\text { nal of Mediaine }\end{array}$ & $42 \%$ & $49 \%$ & $32 \%$ & $52 \%$ & $37 \%$ & $32 \%$ & $56 \%$ & $49 \%$ & $62 \%$ & $0 \%$ & $14 \%$ & $39 \%$ \\
\hline $\begin{array}{l}\text { Phamardogy, } \\
\text { Biochemistryand } \\
\text { Behavior }\end{array}$ & $32 \%$ & $47 \%$ & $0 \%$ & $28 \%$ & $47 \%$ & $8 \%$ & $57 \%$ & $61 \%$ & $32 \%$ & $48 \%$ & $57 \%$ & $38 \%$ \\
\hline $\begin{array}{l}\text { Physiologyand } \\
\text { Behavior }\end{array}$ & $48 \%$ & $49 \%$ & $62 \%$ & $58 \%$ & $47 \%$ & $55 \%$ & $67 \%$ & $58 \%$ & $47 \%$ & $55 \%$ & $69 \%$ & $56 \%$ \\
\hline Totales & $32 \%$ & $43 \%$ & $48 \%$ & $46 \%$ & $46 \%$ & $48 \%$ & $55 \%$ & $53 \%$ & $51 \%$ & $48 \%$ & $45 \%$ & $47 \%$ \\
\hline
\end{tabular}




\section{Cuadro 7}

Artículos de revistas médicas nacionales divididas entre las seis categorías del pe rio do 1986-1996

\begin{tabular}{|l|c|c|c|c|c|c|}
\hline \multicolumn{1}{|c|}{ Nom bre de laRe vis ta } & $\begin{array}{c}\text { Retribución } \\
\text { C1 }\end{array}$ & $\begin{array}{c}\text { Apoyo } \\
\text { Moral } \\
\text { C2 }\end{array}$ & $\begin{array}{c}\text { Auxiliar } \\
\text { C3 }\end{array}$ & $\begin{array}{c}\text { Técnico } \\
\text { C4 }\end{array}$ & $\begin{array}{c}\text { Impulsor } \\
\text { Principal } \\
\text { C5 }\end{array}$ & $\begin{array}{c}\text { Asesor } \\
\text { Acreditado } \\
\text { C6 }\end{array}$ \\
\hline $\begin{array}{l}\text { Ardivos Institutode } \\
\text { Cardidoǵa }\end{array}$ & $14 \%$ & $26 \%$ & $29 \%$ & $18 \%$ & $0 \%$ & $12 \%$ \\
\hline $\begin{array}{l}\text { Ardivos deInvestigadón } \\
\text { Médica }\end{array}$ & $19 \%$ & $19 \%$ & $23 \%$ & $20 \%$ & $8 \%$ & $11 \%$ \\
\hline $\begin{array}{l}\text { BoleténMédicoHospital } \\
\text { InfantildeMéxico }\end{array}$ & $16 \%$ & $21 \%$ & $33 \%$ & $18 \%$ & $0 \%$ & $11 \%$ \\
\hline GactaMédicaMéxico & $42 \%$ & $21 \%$ & $24 \%$ & $0 \%$ & $0 \%$ & $14 \%$ \\
\hline RevistadeInvestigacónClínica & $25 \%$ & $21 \%$ & $24 \%$ & $12 \%$ & $2 \%$ & $17 \%$ \\
\hline RevistaMédica-IMSS & $9 \%$ & $28 \%$ & $39 \%$ & $7 \%$ & $0 \%$ & $17 \%$ \\
\hline SaludPúblicaMéxic & $20 \%$ & $39 \%$ & $15 \%$ & $10 \%$ & $2 \%$ & $14 \%$ \\
\hline \multicolumn{1}{|c|}{ Totales } & $21 \%$ & $25 \%$ & $27 \%$ & $12 \%$ & $2 \%$ & $14 \%$ \\
\hline
\end{tabular}


Presencia de los agradecimientos en los artículos científicos en revistas... 177

Cuadro 8

Artículos de revistas médicas extranjeras divididos entre las seis categorías de agradecimiento

Periodo 1986 - 1996

\begin{tabular}{|c|c|c|c|c|c|c|}
\hline Nombre delaRevista & $\begin{array}{l}\text { Retribución } \\
\text { C1 }\end{array}$ & $\begin{array}{c}\text { Apoyo } \\
\text { Moral } \\
\text { C2 }\end{array}$ & $\begin{array}{c}\text { Auxiliar } \\
\text { C3 }\end{array}$ & $\begin{array}{l}\text { Técnico } \\
\text { C4 }\end{array}$ & $\begin{array}{c}\text { Impulsor } \\
\text { Principal } \\
\text { C5 }\end{array}$ & $\begin{array}{c}\text { Asesor } \\
\text { Acreditado } \\
\text { C6 }\end{array}$ \\
\hline Americanjaurnal ofMedioine & $18 \%$ & $29 \%$ & $13 \%$ & $12 \%$ & $7 \%$ & $20 \%$ \\
\hline Ardhives of Andrdogy & $8 \%$ & $17 \%$ & $9 \%$ & $11 \%$ & $30 \%$ & $25 \%$ \\
\hline Ardives of IntemalMediane & $9 \%$ & $3 \%$ & $10 \%$ & $14 \%$ & $29 \%$ & $36 \%$ \\
\hline BrainRestarch & $8 \%$ & $20 \%$ & $10 \%$ & $6 \%$ & $29 \%$ & $28 \%$ \\
\hline ClinicalResearch & $12 \%$ & $10 \%$ & $19 \%$ & $7 \%$ & $23 \%$ & $29 \%$ \\
\hline Contraception & $8 \%$ & $5 \%$ & $13 \%$ & $7 \%$ & $25 \%$ & $42 \%$ \\
\hline Gastroenterdogy & $14 \%$ & $10 \%$ & $8 \%$ & $15 \%$ & $17 \%$ & $36 \%$ \\
\hline Hepatdogy & $15 \%$ & $11 \%$ & $10 \%$ & $9 \%$ & $28 \%$ & $27 \%$ \\
\hline J. đfBacteridogy & $7 \%$ & $14 \%$ & $19 \%$ & $12 \%$ & $25 \%$ & $25 \%$ \\
\hline J. of Dentistry & $10 \%$ & $5 \%$ & $12 \%$ & $18 \%$ & $33 \%$ & $23 \%$ \\
\hline J. of Natural ProdudsLloydia & $8 \%$ & $12 \%$ & $16 \%$ & $10 \%$ & $23 \%$ & $30 \%$ \\
\hline Joumal of Rhermatdogy & $12 \%$ & $10 \%$ & $13 \%$ & $16 \%$ & $22 \%$ & $26 \%$ \\
\hline Meelical Hypotheses & $4 \%$ & $4 \%$ & $13 \%$ & $9 \%$ & $30 \%$ & $39 \%$ \\
\hline NewEngandJoumal of Meeicine & $13 \%$ & $6 \%$ & $16 \%$ & $6 \%$ & $29 \%$ & $31 \%$ \\
\hline $\begin{array}{l}\text { Phamacology,Biodhemistry } \\
\text { andBehavior }\end{array}$ & $8 \%$ & $15 \%$ & $3 \%$ & $10 \%$ & $29 \%$ & $34 \%$ \\
\hline Physidogyand Behavior & $8 \%$ & $5 \%$ & $12 \%$ & $10 \%$ & $26 \%$ & $38 \%$ \\
\hline Totales & $10 \%$ & $11 \%$ & $12 \%$ & $11 \%$ & $25 \%$ & $31 \%$ \\
\hline
\end{tabular}


178 InvestigaaónBiblicteedógica v. 16 No. 33 julio/ diciembre de 2002

\section{Cuadro 8}

Artículos de revistas médicas extranjeras divididos entre las seis categorías de agradecimiento

Periodo 1986 - 1996

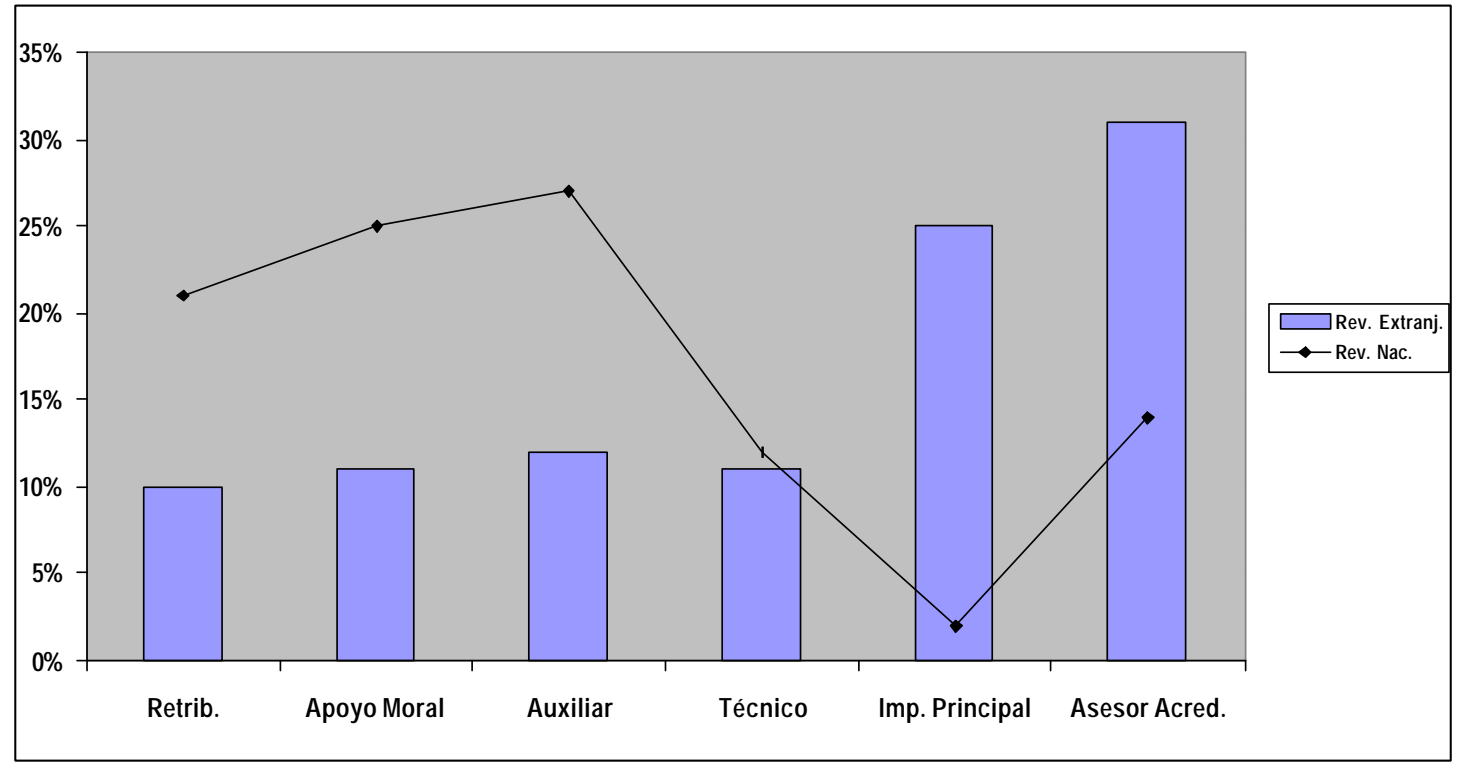

